\title{
An Accidental Arterial Puncture and Anticoagulation after Internal Jugular Vein Catheterization Resulting in Massive Hematoma and Airway Compromise
}

\author{
Kurt Ruetzler,2, ${ }^{1,2 i l l ~ H a u f f e}{ }^{3}$, Tobias Piegeler ${ }^{2}$, Alkadhi Hatem ${ }^{4}$ and Donat R Spahn ${ }^{2}$ \\ ${ }^{1}$ Departments of Outcomes Research and General Anesthesiology, Anesthesiology Institute, Cleveland Clinic, USA \\ ${ }^{2}$ Institute of Anesthesiology, University Hospital Zurich, Switzlerland \\ ${ }^{3}$ Department of Internal Medicine, University Hospital Zurich, Switzlerland \\ ${ }^{4}$ Institute of Radiology, University Hospital Zurich, Switzlerland

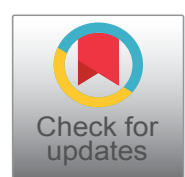 \\ *Corresponding author: Kurt Ruetzler, Departments of Outcomes Research and General Anesthesiology, Anesthesiology \\ Institute, Cleveland Clinic, Cleveland, Ohio, USA; Institute of Anesthesiology, University Hospital Zurich, Zurich, Switzler- \\ land, Tel: 2164074108, Fax: 2164446135, E-mail: kr@or.org
}

\begin{abstract}
Central Venous Catheterization is a common procedure in daily clinical practice. The internal jugular, subclavian and the femoral veins are the most frequently catheterized central veins. Pneumothorax, hematothorax, arterial puncture, hematoma, nerve lesions, damage to the left thoracic duct, and air embolism are among the main mechanical complications. Nowadays, there are two techniques in daily clinical use: the traditional technique and the ultrasound guided technique.

The traditional technique relies on the use of anatomical landmarks, but the failure rate is high- even in experienced hands. Furthermore, several different complications range from mechanical problems (5-19\% of cases) to infections and thrombotic events $(2-26 \%)$. There is compelling evidence that ultrasound-guided CVC insertion via the internal jugular veins is associated with higher success rates and fewer mechanical complications compared with the traditional techniques based on external anatomical landmarks.

We report a clinical case of 76-years-old female patient requiring urgent placement of a central venous catheter and unrecognized arterial puncture and subsequent heparin overdose with nearly fatal outcome.
\end{abstract}

\section{Introduction}

Central venous catheterization (CVC) is widely used in clinical practice for diverse purposes, including invasive hemodynamic monitoring, radiological studies, infusion of drugs that cannot be administered via periph- eral venous access, administration of parenteral nutrition, vascular access in patients whose peripheral veins are difficult to catheterize, and procedures that require access to large-caliber vessels [1]. The central vessels that are most frequently catheterized are the internal jugular, subclavian, and femoral veins.

The traditional CVC insertion technique relies on the use of anatomical landmarks rather than ultrasound guidance. But even in experienced hands, the traditional technique is associated with a high failure rate and several complications ranging from mechanical problems ( $5-19 \%$ of cases) to infections and thrombotic events (2-26\%) [1-4].

Pneumothorax, hematothorax, arterial puncture, hematoma, nerve lesions, damage to the left thoracic duct, and air embolism are among the main mechanical complications [5]. The incidence of complications increase 6-fold after the third insertion attempt [1]. Other risk factors that are known to increase the incidence of complications are an inexperienced operator, the presence of anatomical variants as well as co-existing medical conditions such as clotting disorders, pulmonary emphysema, hypovolemia, or difficulties related to conditions under which the procedure is performed (i.e. an emergency) [5].

Citation: Ruetzler K, Hauffe T, Piegeler T, Hatem A, Spahn DR (2018) An Accidental Arterial Puncture and Anticoagulation after Internal Jugular Vein Catheterization Resulting in Massive Hematoma and Airway Compromise. Int J Anesthetic Anesthesiol 5:069. doi.org/10.23937/2377-4630/1410069 Accepted: July 03, 2018: Published: July 05, 2018

Copyright: (c) 2018 Ruetzler K, et al. This is an open-access article distributed under the terms of the Creative Commons Attribution License, which permits unrestricted use, distribution, and reproduction in any medium, provided the original author and source are credited. 
There is compelling evidence that ultrasound-guided CVC insertion via the internal jugular veins is associated with higher success rate and fewer mechanical complications compared with the traditional techniques based on external anatomical landmarks [5-7].

Written consent to publish this case report was obtained from the patient.

\section{Case Report}

A 76-year-old female patient with chronic obstructive pulmonary disease (COPD) and arterial hypertension was transferred to the emergency department of the university hospital from another peripheral hospital. Patients presented with clinical signs of dyspnea and a newly documented aberrant supraventricular tachycardia in combination with an elevated Troponin Tennon-ST segment elevation myocardial infarction (STEMI) and pneumonia. Immediately after arrival at the emergency department, a coronary angiography was performed, and a severe three coronary arteria disease was diagnosed. Patients was scheduled for coronary artery bypass graft surgery. Laboratory investigations showed elevated white blood cell count (Leucocytes $55 \mathrm{G} / \mathrm{I}$ with lymphocytes $43 \mathrm{G} / \mathrm{l}$ ) with normal coagulation parameters. The elevated blood cell count was interpreted as chronic lymphatic leukemia later on.

The patient was started on therapeutic anticoagulation with unfractionated heparin and infusion rate was adjusted under the guidance of anti-Factor Xa activity (goal: $0.4-0.7 \mathrm{U} / \mathrm{I}$ ). An anti-Xa-activity of 0.7 was reached at a heparin dose of $24.000 \mathrm{IE} / 24 \mathrm{~h}$. During the course of the first day after admittance, the patient developed atrial fibrillation and fever. Due to poor peripheral venous status as well as a presumably longer course of hospitalization, the decision to insert a CVC was made. The on-going heparin-infusion was stopped. Due to the emergent character of the CVC insertion, the procedure had to be performed by the anesthesia team on duty 11 hours after stopping the heparin infusion during the night. The level of anti-Factor Xa activity was $0.04 \mathrm{U} / \mathrm{I}$ at the time of the CVC insertion.

Based on clinical guidelines, the anesthesia team decided to insert a CVC via the right internal jugular vein using an ultrasound-guided technique. The first placement attempt was unsuccessful without aspiration of blood. During the second attempt, obvious venous blood could be aspirated, but insertion of the CVC was impossible. After these two unsuccessful attempts, an experienced attending anesthesiologist took over from the resident initially performing the procedure. The following attempt was successful, venous blood could be aspirated and the CVC could be inserted easily, again using ultrasound-guided technique. After the procedure, a small swelling above the clavicle on the ipsi-lateral side was noted (Figure 1). Suspecting the swelling to be a hematoma, the area was marked, compression on the

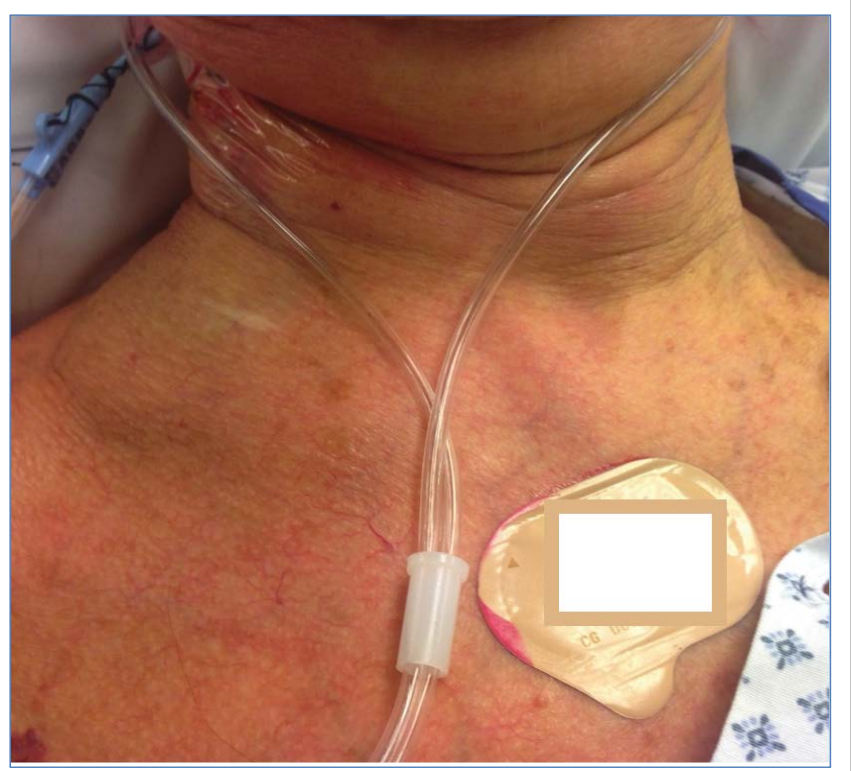

Figure 1: CVC in place with small hematoma above the clavicle.

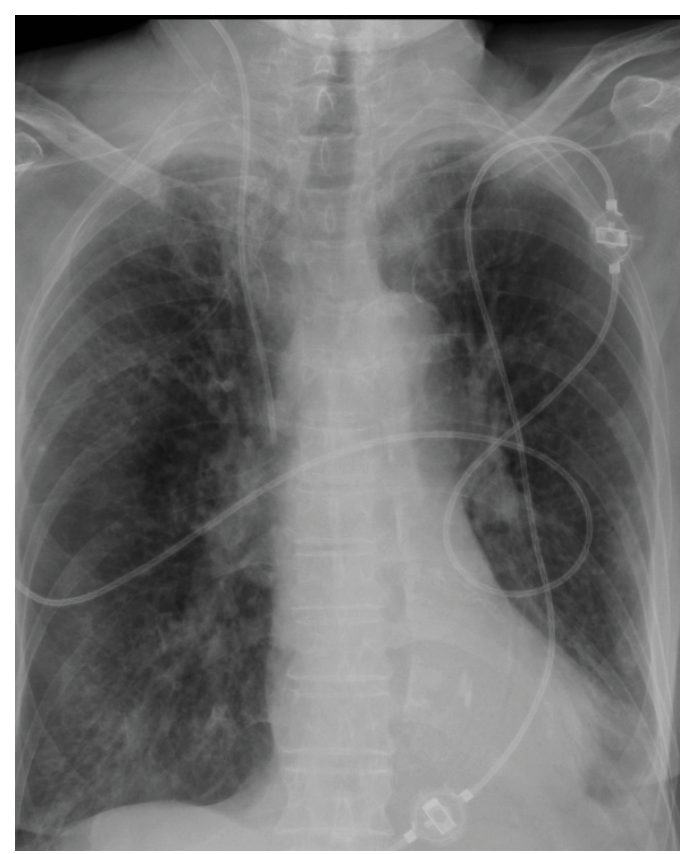

Figure 2: Chest X-Ray indicating correct position of the CVC and increased opacity in the area of suspected hematoma.

right-sided neck was applied using a sand sack and anticoagulation was withheld for approximately 12 hours after the successful puncture in accordance with our local guidelines.

Immediately after the successful CVC insertion a chest X-ray was done, indicating the correct positioning of the catheter right above the right atrium. Additionally, an increased opacity in the area of the right clavicle raised the suspicion of a hematoma (Figure 2).

13 hours after insertion of the CVC, Heparin was re-started with a dose of $25.000 \mathrm{IE} / 24 \mathrm{~h}(=21.7 \mathrm{IE} / \mathrm{kg} / \mathrm{h})$, followed by regular measurements of anti-Xa-activity. After measuring an anti-Factor Xa activity of $1.07 \mathrm{IU} / \mathrm{ml}$ 

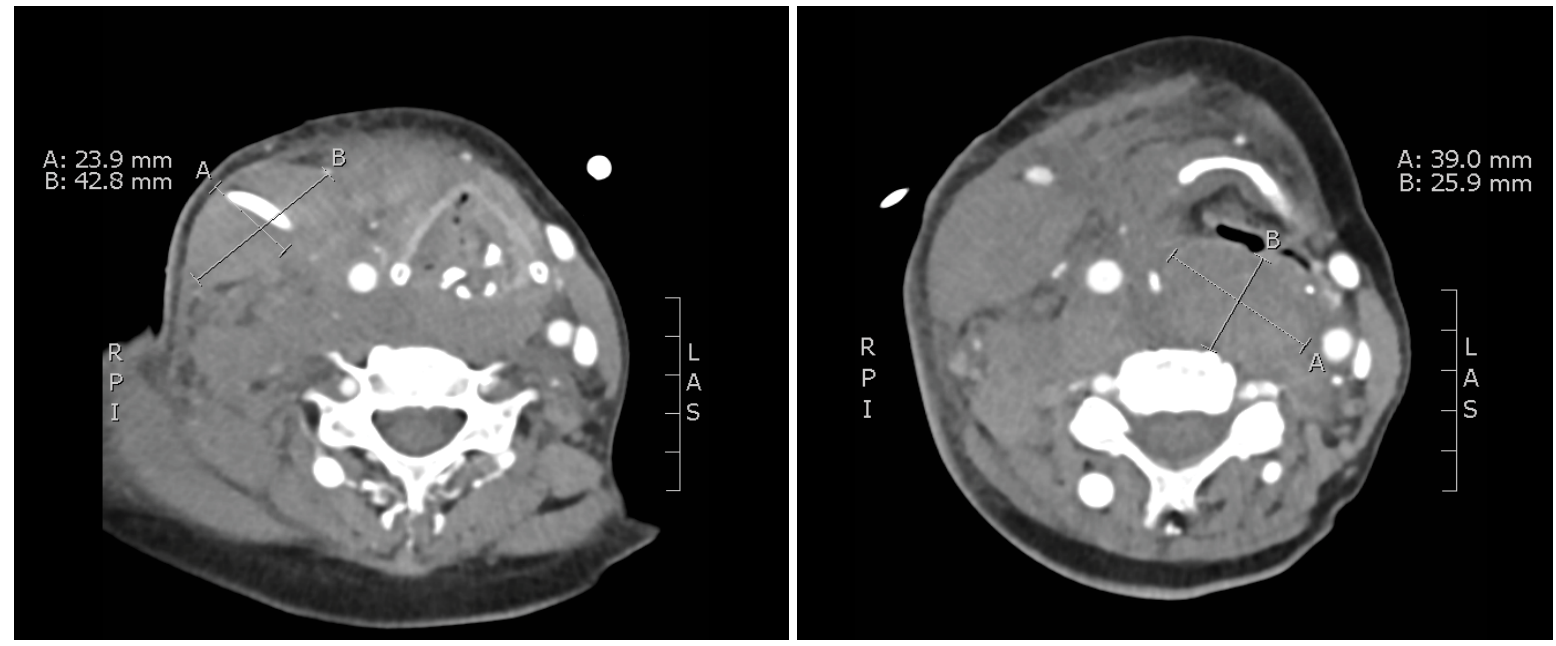

Figure 3: Contrast-enhanced CT images of the neck demonstrating the large hematoma in the sternocleidomastoid muscle (left) and in the retropharyngeal space (right).

Table 1: Summary of the most important findings.

Use of ultrasound-guided CVC technique demonstrate false sense of security.

Although, we could not detect any signs of arterial puncture, a small side branch of an artery was injured.

Anesthesia and intensive care providers must be particulary vigilant in any suspected arterial puncture in the context of subsequent anticoagulation therapy.

five hours after the start of the heparin, the dose was reduced to $22.000 \mathrm{IE} / 24 \mathrm{~h}(=19.1 \mathrm{IE} / \mathrm{kg} / \mathrm{h})$. Another five hours later (18 hours after CVC insertion), an anti-Xa of 1.66 was measured and consequently the heparin dose was reduced to $15.000 \mathrm{IE} / 24 \mathrm{~h}(13 \mathrm{IE} / \mathrm{kg} / \mathrm{h})$ after receiving the results followed by a still elevated anti-Xa activity of $1.05 \mathrm{IU} / \mathrm{ml}$ in the evening of day 2, 36 hours after insertion of the CVC. The dose was again reduced (12.000 IE/24 h). Around 6 hours later (42 hours after CVC insertion), the patient presented with difficulties swallowing and increasing respiratory distress. Clinical examination revealed an enormous swelling of the right-sided neck of about $8 \mathrm{~cm} \times 5 \mathrm{~cm}$. A CT scan of the head and neck was conducted and demonstrated a massive hematoma in the region of the sternocleidomastoid muscle, in the supraclavicular region and in the retropharyngeal space, with compression and displacement of the larynx (Figure 3). By means of an angiography, a small side branch of the thyrocervical artery was identified as a possible source the bleeding (Figure 2). Based on the artery's extremely small diameter, neither surgical nor angiographic intervention appeared as a clinical option.

Due to the rapid clinical deterioration, the patient was finally intubated and transferred to the ICU. The anticoagulation was restarted during the same day. Eight days later, the patient underwent cardiac surgery and could be successfully extubated 2 days later. The postoperative course was complicated with hyperactive delirium and hospital acquired pneumonia resulting in a prolonged stay on ICU and in hospital. After that, successful rehabilitation could be conducted.

\section{Discussion}

In this report we describe a clinical case of massive bleeding after internal jugular vein puncture combined with overwhelming therapeutic anticoagulation.

Bleeding source was probably an accidentally punctured thyrocervical artery or the right jugular vein after multiple punctures in combination with subsequent therapeutic anticoagulation. This is a clinical case reporting an actually unrecognized puncture of a small diameter artery might lead to such dramatic clinical consequences as described.

Bleeding and subsequent hematoma are typical complications of CVC insertion. Bleeding associated with CVC insertion has been reported to range between 0.5 to $1.6 \%$, but rarely seems to be fatal [8]. In several case reports, fatal outcome due to bleeding is most often considered a consequence of inadequate technique or management rather than bleeding diathesis $[9,10]$. The incidence of accidental arterial puncture is approximately $6 \%$ but can significantly be lowered by the use of ultrasound [11]. Even though complication rates in general are lower for the internal jugular vein access compared to other venous access routes, the risk for arterial puncture is higher $[7,12]$. Arterial catheterization has been reported in 0.1 to $1.0 \%$ of CVC insertion procedures [13]. In general, puncture of a branch of the carotid artery is a rare event. Nevertheless, there are already some case reports of injury, fistulas [14] and pseudoaneurysms [15] available.

In summary, a case of unrecognized arterial puncture and subsequent heparin overdose with nearly fatal outcome is presented. Although the hematoma and potential bleeding side effects were recognized after the procedure and the anesthesia team was providing utmost care and precautions, the chain of uncomfortable 
circumstances almost ended in a disaster. In the clinical setting, two independent "errors" were committed: unrecognized arterial puncture and severe overdosing of the anticoagulation therapy. It seems, that arterial puncture occurred despite utmost care and precautions (selection of the right internal jugular vein, physician change after second failed puncture and the use of ultrasound). The severe heparin overdosing should probably be avoided easily due to blood coagulation tests performed on a regular basis. However, comparable single clinical errors repeatedly occur in daily clinical practice. In this case report, the unfortunate combination almost leads to catastrophic clinical consequences (Table 1 ).

As a conclusion, the use of ultrasound guided CVC technique demonstrates a "false sense of security" and avoidance of any serious complications. However, in the case of any suspected arterial puncture in the context of subsequent anticoagulation therapy, anesthesia and intensive care providers must be particularly vigilant.

\section{References}

1. McGee DC, Gould MK (2003) Preventing complications of central venous catheterization. N Engl J Med 348: 11231133.

2. Mansfield PF, Hohn DC, Fornage BD, Gregurich MA, Ota DM (1994) Complications and failures of subclavian-vein catheterization. N Engl J Med 331: 1735-1738.

3. Merrer J, De Jonghe B, Golliot F, Lefrant JY, Raffy B, et al. (2001) Complications of femoral and subclavian venous catheterization in critically ill patients: A randomized controlled trial. JAMA 286: 700-707.

4. Veenstra DL, Saint S, Saha S, Lumley T, Sullivan SD (1999) Efficacy of antiseptic-impregnated central venous catheters in preventing catheter-related bloodstream infection: A meta-analysis. JAMA 281: 261-267.

5. Vezzani A, Manca T, Vercelli A, Braghieri A, Magnacavallo A (2013) Ultrasonography as a guide during vascular access procedures and in the diagnosis of complications. $J$
Ultrasound 16: 161-170.

6. Frykholm P, Pikwer A, Hammarskjöld F, Larsson AT, Lindgren S, et al. (2014) Clinical guidelines on central venous catheterisation. Swedish society of anaesthesiology and intensive care medicine. Acta Anaesthesiol Scand 58: 508524.

7. Ruesch S, Walder B, Tramèr MR (2002) Complications of central venous catheters: Internal jugular versus subclavian access--a systematic review. Crit Care Med 30: 454-460.

8. Eisen LA, Narasimhan M, Berger JS, Mayo PH, Rosen MJ, et al. (2006) Mechanical complications of central venous catheters. J Intensive Care Med 21: 40-46.

9. Domino KB, Bowdle TA, Posner KL, Spitellie PH, Lee LA, et al. (2004) Injuries and liability related to central vascular catheters: A closed claims analysis. Anesthesiology 100: 1411-1418.

10. Hove LD, Steinmetz J, Christoffersen JK, Møller A, Nielsen $\mathrm{J}$, et al. (2007) Analysis of deaths related to anesthesia in the period 1996-2004 from closed claims registered by the Danish Patient Insurance Association. Anesthesiology 106: 675-680.

11. Reuber M, Dunkley LA, Turton EP, Bell MD, Bamford JM (2002) Stroke after internal jugular venous cannulation. Acta Neurol Scand 105: 235-239.

12. Schummer W, Schummer C, Rose N, Niesen WD, Sakka SG (2007) Mechanical complications and malpositions of central venous cannulations by experienced operators: A prospective study of 1794 catheterizations in critically ill patients. Intensive Care Med 33: 1055-1059.

13. Kusminsky RE (2007) Complications of central venous catheterization. J Am Coll Surg 204: 681-696.

14. Zachariah PP, Unni VN, Kurian G, Nair RR, Mathew A (2014) Thyrocervical artery - jugular fistula following internal jugular venous catheterization. Indian J Nephrol 24: 178-180.

15. Mazzei V, Benvenuto D, Gagliardi M, Guarracini S, Di Mauro M (2011) Thyrocervical trunk pseudoaneurysm following central venous catheterization. J Card Surg 26: 617-618. 